\title{
Chronique bibliographique : l'objet diaspora en questions
}

\section{Stéphane Dufoix}

\section{(2) OpenEdition}

12 Journals

\section{Édition électronique}

URL : http://journals.openedition.org/conflits/495

DOI : $10.4000 /$ conflits.495

ISSN : $1777-5345$

Éditeur :

CCLS - Centre d'études sur les conflits lilberté et sécurité, L'Harmattan

Édition imprimée

Date de publication : 15 mai 1999

ISSN : 1157-996X

Référence électronique

Stéphane Dufoix, "Chronique bibliographique : l'objet diaspora en questions », Cultures \& Conflits [En ligne], 33-34 | printemps-été 1999, mis en ligne le 16 mars 2006, consulté le 30 mars 2021. URL

http://journals.openedition.org/conflits/495 ; DOI : https://doi.org/10.4000/conflits.495

Ce document a été généré automatiquement le 30 mars 2021.

Creative Commons License 


\title{
Chronique bibliographique : l'objet diaspora en questions
}

\author{
Stéphane Dufoix
}

1 Georges Prévélakis, Les réseaux des diasporas, Paris-Nicosie, L'Harmattan-KYKEM, 1996. Robin Cohen, Global diasporas. An introduction, Londres, UCL Press, coll. Global diasporas, 1997. Nicholas van Hear, New diasporas. The mass exodus, dispersal and regrouping of migrant communities, Londres, UCL Press, coll. Global diasporas, 1998.

2 «J'y consens, leur dis-je, car je ne dispute jamais du nom, pourvu qu'on m'avertisse du sens qu'on lui donne » Pascal, Première provinciale

Depuis une dizaine d'années, l'utilisation scientifique du terme de « diaspora » a connu une fantastique inflation dans le domaine des sciences sociales. Auparavant réduite à quelques «peuples » (Juifs, Arméniens, puis Indiens et Chinois), elle a progressivement pris de l'extension jusqu'à englober à l'heure actuelle plus d'une cinquantaine de populations. La notion de diaspora est au cœur de nombre d'interrogations récentes ou renouvelées récemment dans le champ des sciences sociales : le postmodernisme ${ }^{1}$, la mondialisation $^{2}$ et le nationalisme ${ }^{3}$. Si la recherche sur ce thème en France demeure moins importante que dans les pays anglo-saxons, elle a récemment connu un grand développement ${ }^{4}$. Les trois ouvrages que nous avons choisis représentent, selon nous, les recherches les plus importantes des trois dernières années sur le thème de la diaspora. Ils présentent aussi la particularité de montrer - bien que de façon non exhaustive l'étendue des types de recherche mettant en avant le terme de "diaspora »: les monographies et leurs " produit de synthèse » (Prévélakis), l'ouvrage à visée théorique (Cohen) et la recherche comparative de terrain (van Hear). Ils caractérisent aussi les limites du domaine scientifique de l'étude des diasporas puisque Cohen ne mentionne pas Prévélakis dans sa bibliographie, de même que van Hear ne cite ni le livre de Cohen ni celui de Prévélakis. La structure et le contenu des ouvrages sont très différents. Les réseaux des diasporas rassemble 31 interventions faites lors d'un colloque tenu à Chypre au printemps 1993 sur le thème générique de la diaspora. Cinq de ces chapitres (écrits par Jean Gottmann, Georges Prévélakis, Gabriel Sheffer, Stanley Brunn et Paul Claval) ont pour objet la discussion théorique de la notion, tandis que les 26 autres 
s'attachent à l'étude d'une population particulière ${ }^{5}$ ou d'un pays d'accueil particulier ${ }^{6}$. L'ouvrage de van Hear se distingue des deux autres par l'intégration de la " diaspora » dans un modèle théorique plus large visant à la prise en compte de l'ensemble des mouvements migratoires. Il étudie, dans cinq parties du monde (Afrique, Europe balkanique, Asie, Amérique et Moyen-Orient), dix crises migratoires caractérisées par l'exode - ou l'expulsion - massif de "communautés migrantes » hors du pays où elles vivaient. Le grand mérite de Nicholas van Hear est ici de nous initier à des épisodes dont certains sont en partie balisés - l'expulsion des Asiatiques ougandais en 1972, des Turcs de Bulgarie en 1989 et des Albanais de Grèce en 1990-94 - mais dont la plupart sont presque totalement méconnus en France: l'exode des Africains de l'Ouest du Nigeria en 1983, des Palestiniens du Koweït en 1990-92, des Yéménites d'Arabie Saoudite en 1990-92, des Bangladeshis musulmans de Birmanie - le Myanmar depuis 1988 - , des Népalais du Bouthan en 1991-92, des Haïtiens de République dominicaine en 1991 et enfin les Mexicains des États-Unis en 1954. Jusqu'à présent, la recherche scientifique sur les diasporas a essentiellement pris corps dans les disciplines de la géographie, de l'histoire, ainsi que dans celles de la science politique et de la sociologie dans leur version internationale, à quelques exceptions françaises près ${ }^{7}$. La multiplication des études a eu pour conséquence l'accumulation de connaissances sur l'ampleur et la destination des flux migratoires en provenance de nombreux pays, ainsi que sur la création et l'organisation de communautés nationales à l'étranger. Pourtant, force est de constater que ce champ de recherche bute sur le problème fondamental de la définition, et ce des deux manières identifiées par Durkheim dans Les règles de la méthode sociologique : par absence de rupture avec le sens commun et par manque de rigueur dans l'étendue de la définition, c'est-à-dire dans sa construction. Comme l'a montré la récente conférence d'Howard Gilman sur les diasporas et le transnationalisme, il est possible de consacrer trois jours à ce thème sans presque jamais se poser la question de la définition car, comme il le fut affirmé, « tout le monde s'entend sur le sens du mot diaspora ". Notre propos n'est pas ici de présenter ces ouvrages dans l'exhaustivité de leurs développements. D'ailleurs, leur contenu descriptif laisse peu de place à la critique. La présentation des "communautés » étrangères que l'on trouve dans les différentes études du livre de Prévélakis, dans les chapitres 2 à 6 de Cohen ainsi que dans les chapitres 3 et 4 de van Hear est toujours précise et sérieuse. Ce n'est pas cette partie de leur travail qui fait l'objet de notre analyse, mais bien plutôt l'utilisation qu'ils font de l'objet scientifique « diaspora » et de la méthode qu'ils emploient pour en circonscrire le sens et en entreprendre la recherche. A travers eux, nous tenterons de montrer de façon critique l'état de la recherche dans ce domaine. Selon nous, trois illusions enferment pour l'instant l'étude scientifique des diasporas :

- l'illusion de la substance. Elle consiste, selon l'expression de Ludwig Wittgenstein, en "la recherche d'une substance qui réponde à un substantif». C'est alors la dénomination qui vient en premier par rapport au réel. La définition n'en est pas nécessaire puisqu'elle relève la plupart du temps du sens commun: on sait d'ores et déjà ce qu'est une diaspora et la recherche implique de confronter la réalité des peuples dispersés à l'aune du modèle premier de manière à mettre en évidence quelles sont les « vraies » diasporas. De la sorte, on peut les classer en diasporas classiques, historiques, nouvelles ou modernes selon leur degré de proximité avec l'archétype ${ }^{8}$. Cette illusion est au principe de l'exclusion de nombreuses populations du champ définitionnel de la 
diaspora, dont on trouve un exemple dans l'article de Louis-André Sanguin : « Un usage abusif et maximaliste du concept consiste à considérer toute population immigrante en provenance d'un pays tiers comme une diaspora. Ainsi les 36000 Français et les 32.000 Britanniques de Montréal seraient des diasporas! Or, "tel n'est pas le cas puisqu'il s'agit de personnes provenant de pays riches, démocratiques et non surpeuplés qui ont émigré sur une base strictement individuelle et volontaire afin de réaliser un projet personnel ou professionnel ». (RD, « Les diasporas et leurs trajectoires dans les grandes métropoles canadiennes : le cas de Montréal », p. 410-411, souligné par nous).

- l'illusion communautaire. Elle suppose que la diaspora correspond à l'ensemble des membres dispersés de la population considérée. A aucun moment n'est mise en cause la règle de réification consistant à totalement passer sous silence la structuration spatiale et temporelle desdites "communautés transnationales». Les quantifications entreprises pour mesurer l'ampleur d'une diaspora prennent en compte de façon indifférente tous les pays concernés par la présence étrangère en question ainsi que l'époque de leur migration. Sans même mettre l'accent sur le fait que certaines de ces quantifications restent muettes sur l'inclusion ou non des générations issues des migrants, cette conception de la diaspora est homogénéisante. Elle oublie qu'une communauté n'existe comme telle que par sa construction sociale et par la mise en place d'un travail de représentation du groupe ${ }^{9}$.

- l'illusion fixiste. Souvent implicite, elle postule néanmoins l'a-historicité, la permanence de ces communautés transnationales au travers des années ou des siècles si cela se présente - sans modification fondamentale de leur organisation, comme si leur existence était désormais inscrite dans le cours des choses. Ceci est particulièrement visible dans le cas de la diaspora juive, exemple paradigmatique, dont la continuité est présumée sans faille de la destruction du Temple de Jérusalem en 70 après Jésus-Christ au moins jusqu'à la fondation de l'État d'Israël (Gorny, «Center and periphery. Changing relations between the State of Israël and the Jewish diaspora », in Prévélakis, p. 47-52). Comme dans l'ensemble des études portant sur les phénomènes migratoires, le comparatisme est revendiqué dans la recherche sur les diasporas comme le moyen de parvenir à une meilleure connaissance du phénomène. Pourtant, si van Hear consacre plusieurs pages à une analyse comparée systématique des résultats accumulés sur les dix crises migratoires qu'il a choisies, tant $C$ Chen ${ }^{10}$ que Prévélakis ont tendance à pratiquer un comparatisme de convenance, plus caractérisé par la juxtaposition de cas que par une réelle confrontation entre les exemples choisis. C'est dans Les réseaux des diasporas que cette tendance au faux comparatisme est la plus visible, confortée par la logique même de juxtaposition qui est au principe de tout ouvrage collectif. Si l'on se reporte à la triple classification des modes de comparaison en matière d'études migratoires, élaborée par Nancy Green, il s'avère que fort peu d'articles peuvent prétendre au comparatisme ${ }^{11}$. Le modèle convergent, qui étudie plusieurs populations sur le territoire d'un seul et même pays d'accueil, n'est représenté que par trois contributions sur la géographie des diasporas arménienne, juive et grecque de l'ex-URSS (p. 93-114), l'immigration en milieu bilingue à Bruxelles (p. 399-408) et les trajectoires des diasporas à Montréal (p. 409-432). Le modèle divergent, s'attachant à la comparaison d'une même population dans au moins deux pays d'accueil, est totalement absent. Le modèle linéaire, confrontant l'émigré au pays d'accueil à ce qu'il était dans le pays d'origine, est implicitement présent dans tous les 
articles se concentrant sur une seule population, mais il n'est jamais systématisé. De la sorte, le comparatisme de RD - volontiers incantatoire - se limite à la juxtaposition d'études « nationales » dont certaines se « contentent » de signaler les vagues de départ et d'additionner la population des "communautés" censées composer la diaspora, confortant ainsi l'idée que cette dernière correspond exactement à la somme des dispersés. Un exemple typique de cette posture est l'article de Nurit Kliot, «Asian diaspora's role in international politics : features and evolution » (p. 69-80), dont le contenu est de surcroît totalement en porte-à-faux avec un titre impliquant a contrario une signification collective bien plus importante, dans le cas de populations indiennes pour lesquelles d'autres travaux ont commencé à montrer tout l'intérêt de l'application d'un modèle divergent ${ }^{12}$. En ce sens, Comment utiliser scientifiquement un terme dont le sens est historiquement et culturellement connoté ? Depuis les débuts de l'utilisation scientifique de la notion de diaspora, elle reste marquée par l'expérience juive à tel point que beaucoup considèrent que cette dernière est l'expérience diasporique par excellence et que tout autre usage du terme ne peut être que métaphorique. Il est significatif de ce marquage que nombre d'articles, et notamment celui de Jean Gottman dans Les réseaux des diasporas ( $\mathrm{La}$ généralisation des diasporas et ses conséquences »), s'attardent avant tout sur le mot, retraçant ses origines grecques puis son utilisation quasi exclusive pour le peuple juif, pour ne considérer le "phénomène " que par la suite, non sans le surcharger d'un sens réificateur avant même tout effort de définition : «Le phénomène de diaspora s'est généralisé » (p. 22). Tout se passe comme si la définition même du phénomène était consubstantielle du mot « diaspora ». C'est alors le mot qui précède l'idée et justifie qu'il soit peu nécessaire de s'interroger plus avant sur le sens qu'on lui donne puisqu'il se trouve intégralement contenu dans l'exemple de la dispersion juive. Pourtant, les étapes apparemment élémentaires d'une telle affirmation sont manquantes. Non seulement, l'histoire de l'apparition du mot grec dans les autres langues n'a semble-t-il jamais été faite, mais on n'a pas suffisamment pris en compte l'exacte signification des dénominations indigènes du phénomène, ainsi que les correspondances entre elles ${ }^{13}$. Quel point commun compréhensif y a-t-il entre "diaspora » en hébreu et le spiurk arménien? D’autant qu'un élément fondamental devrait inciter à bien plus de prudence les chercheurs soucieux de fonder l'étude des diasporas sur l'exemple juif. Le sens même de " diaspora » en hébreu n'est pas fixé, puisque, à en croire Richard Marienstras, il peut être distingué de "galut» (exil) pour ne désigner que l'ensemble des communautés juives quand il existe un centre politique, contrairement à "galut» impliquant l'absence de centre politique ${ }^{14}$. L'article de Yosef Gorny reconnaît d'ailleurs l'existence d'un tel débat après la fondation de l'État d'Israël. On rencontre la même historicité du terme et les mêmes conflits sémantiques dans le cas arménien, « spiurk » apparaissant en 1920 pour ne s'imposer qu'après la seconde guerre mondiale. En dépit des obstacles que nous venons de signaler, l'entreprise de définition de ce que serait une diaspora demeure étonnamment rudimentaire, se contentant souvent d'une extension plus ou moins maitrisée du cas juif. La diaspora devient alors synonyme de communauté ethnique à l'étranger. L'étude de Nicholas van Hear se contente de ce qu'il appelle une «perspective lâche » (loose perspective) sur la diaspora (p.6) : peut être appelée ainsi toute population satisfaisant au moins trois critères : une dispersion sur le territoire d'au moins deux pays d'accueil à partir d'un pays d'origine ; une présence persistante à l'étranger, bien qu'elle soit compatible avec une certaine circulation entre les pays d'origine et d'accueil ; enfin, l'existence d'échanges (sociaux, politiques, économiques 
ou culturels) entre les populations composant la diaspora. Ces trois éléments sont présents dans d'autres définitions, mais rarement ensemble. Le premier se retrouve chez Robin Cohen (p.23), ainsi que dans la conception de la diaspora élaborée par Emmanuel Ma Mung et qui sert de trame à son article dans RD (« Non-lieu et utopie : la diaspora chinoise et le territoire», p. 205-2145). Le second est explicitement mis en avant comme aspect constitutif par Georges Prévélakis ("Les réseaux des diasporas ", p. 29-34) mais entre dans le cadre conceptuel d'autres auteurs comme Richard Marienstras. Enfin, le troisième fait également partie de la problématique de Ma Mung. L'introduction d'un élément temporel dans la définition d'une diaspora n'est pas sans soulever trois difficultés importantes que l'on retrouve tout au long des études prises en compte dans cet article. Premièrement, elle impose l'idée d'un seuil au-delà duquel existent les diasporas, ce qui pose le même problème que l'élément quantitatif suggéré par Yves Lacoste, pour qui une diaspora est la dispersion de plus de la moitié d'un peuple $^{16}$ : au bout de combien d'années? A partir de combien de personnes ? Comment établir la limite pertinente ? D'où proviennent ces mystérieuses frontières temporelles et statistiques au-delà desquelles la simple dispersion devient diaspor $\mathrm{a}^{17}$ ? Deuxièmement, la prise en compte de cette variable prédominante présuppose une analyse uniquement a posteriori, liée au succès ou à l'échec de ces populations dispersées à se constituer - comment?; nous ne l'apprenons pas - en diaspora, touchant ainsi à une évidente pérennité. Outre l'aspect très critiquable d'une telle démarche fondée sur le résultat des processus et non sur les processus eux-mêmes, il lui échappe nécessairement toute l'épaisseur de ce qui est en train de se faire pour ne retenir que le déjà-réussi ou le déjà-échoué. Enfin, le postulat de l'existence de seuils implique un certain dynamisme dans la définition - la "diasporisation " plus que la « diaspora »- alors que, par ailleurs, les problématiques incluant le facteur temporel ou quantitatif ont la plupart du temps une vision spontanéiste et réificatrice de la diaspora dans laquelle diaspora est simplement un synonyme de dispersion. Malgré toutes les précautions qu'il prend pour lier la diaspora à l'existence de réseaux qui en fondent l'existence et permettent les échanges par opposition au simple transnationalisme qui ne suppose qu'une double allégeance, au pays d'accueil et au pays d'origine (p. 249), Nicholas van Hear De même, c'est une conception à la fois maximaliste et spontanée de la diaspora qui prévaut dans RD, à l'image de la phrase de Jean Gottmann selon laquelle « il devient difficile aujourd'hui de trouver une nation qui n'ait pas sa diaspora, c'est-àdire qui n'ait pas une partie souvent importante de son peuple, dispersée en dehors des frontières de l'État national » (p. 22). Stanley Brunn étend la notion jusqu'à l'extrême et de fait la rend totalement inutile - en définissant la diaspora comme «un mouvement de population ou un groupe déplacé d'un lieu pour prendre résidence dans un autre pays", se trouvant ainsi comprendre également les touristes et les participants aux Jeux Olympiques! ("The internationalization of diasporas in a shrinking world», p. 259-272). Les monographies incluses présentent, elles, trois visages différents : 1 ) Certains auteurs conservent une définition de sens commun où la diaspora équivaut à l'ensemble des membres d'une population nationale dispersés sur le territoire d'autres pays, ou transforment légèrement cette approche pour la limiter à un seul territoire. Dans ce dernier cas, "diaspora" équivaut à "ethnie d'origine étrangère » (Gérard-François Dumont, « Diasporas et valeurs républicaines en France, p. 355-372). 2) D'autres forgent une définition personnelle de la diaspora pour tenter de la démontrer par la description monographique. Cependant, ces tentatives s'avèrent peu concluantes. Soit l'absence de comparaison avec d'autres études de cas tend à n'en 
faire que des extrapolations généralisantes à partir d'un seul cas, soit elles présentent de grandes difficultés théoriques et empiriques comme celle qui définit les diasporas comme des «communautés ethniques trouvant leur origine dans la migration, qui se sont socialement diversifiées et prennent part à l'économie nationale, mais qui sont demeurées culturellement fermées" (Jeroen Doomernik et Hans van Amersfoort, "Immigrant community or diaspora? Turkish immigrants in the Netherlands", p. 387-398). En l'occurrence, tant la prise en compte comme un tout de ces " communautés ethniques » que la complexité de la mesure de la diversification sociale et de la fermeture culturelle - ainsi que du degré d'inférence qu'il faut leur accorder interdisent à ces définitions de devenir des points de départ. 3) Enfin, quelques rares contributions se réclament explicitement d'une définition de la diaspora élaborée par un autre auteur.

7 Si Paul Claval estime dans sa conclusion que « les participants ont accepté l'idéal-type de la diaspora présenté par Gabi Sheffer » («Diasporas and politics, an overview », p. 433-444), nous sommes en fait d'avis que RD se partage entre deux conceptions assez éloignées de la diaspora, celle de Gabriel Sheffer et celle d'Emmanuel Ma Mung. La première ("Whither the study of ethnic diasporas? Some theoretical, definitional, analytical and comparative considerations ", p. 37-46) reprend les analyses élaborées dix ans plus tôt ${ }^{18}$. L'appellation de "diaspora » est justifiée quand les "communautés » présentent les éléments objectifs suivants : elles sont des entités politiques et sociales transnationales; elles résultent d'une migration volontaire ou contrainte vers un ou plusieurs pays d'accueil ; leurs membres résident de façon permanente dans les pays d'accueil ; elles y constituent des minorités; elles témoignent d'une identité ethnique explicite; elles créent et maintiennent des organisations communautaires conséquentes; elles font preuve de solidarité avec d'autres membres de la communauté et donc une certaine cohérence culturelle et sociale; elles mettent en œuvre, par l'intermédiaire des organisations communautaires, des activités culturelles, sociales, politiques et économiques; elles maintiennent des échanges culturels, sociaux, politiques et économiques avec le pays d'origine, que ce dernier soit un Etat ou une communauté sur un territoire considéré comme leur patrie ; elles créent des réseaux transnationaux pour faciliter l'échange de ressources significatives avec la patrie ou avec d'autres communautés dans d'autres pays d'accueil; enfin, elles possèdent un potentiel de coopération ou de conflit avec le pays d'accueil et le pays d'origine (p. 39). La seconde conception est beaucoup plus simple, mais aussi plus fondée sur les processus que sur des indicateurs statiques. Selon Emmanuel Ma Mung, deux caractères morphologiques objectifs définissent la diaspora: la multipolarité de la migration et l'interpolarité des relations avec le pays d'origine et entre les différents pôles de la migration $^{19}$. Essentiellement fondée sur l'exemple chinois, elle n'en constitue pas moins à notre sens une base de départ plus riche de perspectives que celle de Sheffer car plus articulée sur la logique des processus que sur celle des critères typologiques. Ces articles théoriques présentent certes une plus grande réflexion sur le phénomène, mais elle est dissociée de la réalité, uniquement centrée sur la volonté de découper une partie du réel à partir d'une définition préalable plutôt que de pratiquer un aller-retour entre l'élaboration théorique et l'accumulation de données empiriques étayant et enrichissant en retour la montée en généralité. En revanche, Robin Cohen prend ce problème de définition à bras-le-corps. Global diasporas est la première tentative générale de clarification de la notion de diaspora qui soit entreprise par un seul auteur à l'échelle d'un ouvrage (l'ouvrage de Sheffer en 1986 était collectif). A cet égard, elle 
est la référence fondamentale et l'indispensable point de départ de toute réflexion sur le sujet. La plupart des précédentes définitions ou catégorisations - et les deux sont nombreuses - de la diaspora relèvent de deux ensembles bien distincts : définition de la diaspora par énumération des conditions requises à l'obtention de cette qualification par une population (Sheffer) ou détermination de sous-divisions à l'intérieur de la catégorie générale de "diaspora » qui n'était pas discutée. Les qualificatifs accolés à " diaspora » sont légion: ethnique, émergente (incipient), nourricière/matrice, d'asile, dormante, mobilisée/prolétaire etc.. Comme Gabriel Sheffer avant lui ${ }^{20}$, Robin Cohen, lui, lie les deux en une seule démarche qui, de surcroît - contrairement à Sheffer parvient à s'affranchir de la seule théorie pour proposer un ouvrage combinant brillamment la réflexion critique et une grande connaissance de nombreuses « diasporas» (juive, africaine, arménienne, indienne, britannique, chinoise, libanaise, sikh et caraïbe). Cohen part du principe que l'analyse scientifique de la diaspora doit s'affranchir à la fois de l'expérience juive et du modèle « catastrophique » pour prendre en compte des phénomènes ressemblants, mais auxquels la dénomination de diaspora n'a pas nécessairement été appliquée jusqu'alors. Pour ce faire, il dessine, en prenant appui plus sur William Safran ${ }^{21}$ que sur Gabriel Sheffer, une liste de neuf caractéristiques communes (common features) des diasporas que l'on peut résumer ainsi : 1) dispersion souvent traumatique sur au moins deux territoires étrangers ou 2) expansion territoriale dans un but de conquête, de travail ou de commerce; 3) existence d'une mémoire collective du pays d'origine ; 4) idéalisation du pays natal et engagement collectif envers son maintien ou sa création; 5) développement d'un mouvement de retour collectivement approuvé; 6) forte conscience ethnique de groupe ; 7) rapport conflictuel avec les sociétés d'accueil ; 8) empathie et solidarité avec les membres du groupe ethnique installés sur d'autres territoires; et 9) la possibilité de développer un sens créatif dans des pays tolérants (p. 26). Sur cette base, il différencie cinq types de diasporas par l'ajout d'un adjectif qualificatif : victimes (Juifs, Arméniens et Africains), laborieuses (Indiens), impériales (Britanniques), commerciales (Chinois et Libanais) et enfin culturelles (Caraïbes), auxquelles sont ensuite consacrées quatre chapitres. Les trois chapitres restants concernent le rapport des diasporas à la patrie, le rapport entre globalisation et diasporas, et un chapitre de conclusion. Etant donné sa richesse, il est difficile de livrer ici une critique très fouillée de la thèse de Cohen. Nous nous contenterons de souligner quelques aspects des points communs et de son originalité par rapport à d'autres études. Tout d'abord, l'étude présente deux qualités indéniables :

- Tant sa liste de critères que sa typologie nous semblent plus pertinents que celles de Sheffer en particulier par l'importance qu'il accorde à la question de l'identité et à sa construction, et par la mise en place d'une typologie ne se fondant pas sur la temporalité, mais sur de réels types-idéaux.

- Bien qu'il ne le mentionne que brièvement (p. x), Cohen semble très attentif aux limites de sa démarche. Il reconnaît que son modèle est trop rigide pour pouvoir appréhender la réalité des formes et de l'évolution des diasporas. Cependant, malgré les qualités ci-dessus, malgré les précautions prises, l'analyse de Cohen souffre de deux limites principales:

- La diaspora demeure une notion figée, en particulier parce qu'elle n'est jamais définie en référence à ses membres eux-mêmes. Elle reste une entité qui les dépasse à laquelle ils semblent voués à appartenir dès lors qu'ils sont dispersés à partir de leur territoire d'origine. La capacité d'identification que Cohen met souvent en avant n'est pas utilisée 
pour la construction même de la cohérence du groupe. Pour qu'il y ait mémoire collective, il ne suffit pas de mémoires individuelles: il faut des groupes, des organisations, des porte-parole.

- Ensuite, la typologie qu'il propose n'est en elle-même pas cohérente. Alors que les quatre premiers types sont définis par leur caractéristique d'origine (une catastrophe, le commerce, le travail, l'impérialisme), la diaspora culturelle possède une définition à part. Plus exactement, elle n'en a pas : à aucun moment n'est explicitement mis en avant le sens de « diaspora culturelle ». Cette catégorie n'est explorée (p. 127-153) qu'au travers des postulats post-modernistes sur la mouvance des identités et leur hybridité, ainsi qu'à travers l'exemple caraïbe. Dans ce dernier cas, Cohen utilise la technique du syllogisme: pour les post-modernistes, les peuples caraïbes sont une diaspora culturelle, une culture itinérante; pour que cela entre dans la typologie, il faudrait qu'ils satisfassent aux critères d'affirmation d'une identité africaine, d'intérêt pour le retour, de présence de produits culturels hybrides entre l'Afrique, la Caraïbe et les pays d'accueil, enfin l'existence d'une conscience commune de cette identité diasporique. On peut en trouver des exemples, donc il s'agit d'une diaspora culturelle. Pourtant, aucun des problèmes n'a été réglé : qui en fait partie? Comment s'est historiquement mise en place cette diaspora? Surtout, si les diasporas ne sont pas uniquement déterminées par leur origine, si elles peuvent se former après, pourquoi la typologie demeure-t-elle essentiellement fondée sur ce critère? La récente critique, par Floya Anthias, de la notion de diaspora, et notamment de l'ouvrage de Robin Cohen, pointe certaines difficultés également mises en évidence ici, mais elle ne remet pas en cause l'objectivation de la diaspora comme sujet collectif sui generis ${ }^{22}$. La limitation qu'elle en fait à un outil de typologie descriptive et à une condition sociale ne permet pas de dépasser la nécessité de rendre compte de ce que serait une diaspora par rapport à d'autres modes d'organisation sociale d'une population émigrée. Si la dimension sexuée est indéniablement importante et heuristique pour l'étude des populations émigrées, comme l'avait précédemment montré Diana Kay à propos de l'exil chilien en Ecosse ${ }^{23}$, elle ne peut pas être une première étape dans une démarche de définition. En effet, sur la base des recherches incluses dans RD et de celle de Robin Cohen, il est évident que l'étude des diasporas s'est engagée dans la voie de modèles statiques ne permettant pas de passage vers la diaspora ni de celle-là vers autre chose. New Diasporas est incontestablement, des trois ouvrages, le plus éloigné des études classiques sur les diasporas puisque la majeure partie de ses développements n'y sont pas consacrés. En revanche, le modèle qu'il élabore pour l'analyse des mouvements migratoires (p. 40-47) et qui comprend la diaspora, est très novateur et riche de perspectives. Cherchant à perpétuer les récentes analyses sociologiques - notamment celles d'Anthony Richmond ${ }^{24}$ - visant à brouiller la frontière trop nette entre migrations volontaires et forcées, il met en place un double cadre d'analyse. Sur un premier axe se distribuent les éléments de choix et de contrainte selon un continuum allant de la migration contrainte avec peu de choix, peu d'options (souvent vivre ou mourir), à des migrations de plus en plus volontaires, c'est-à-dire dont les degrés de choix (quand partir, où) augmentent. Sur un second axe, van Hear procède à une «désagrégation des mouvements migratoires» (p. 41) pour en extraire les composantes. Il en résulte une matrice structurelle des cinq sens migratoires: vers l'extérieur (outward - sortie du pays d'origine) ; vers l'intérieur (inward - entrée sur le territoire d'un pays); retour (return) ; réémigration (onward - d'un premier pays d'accueil vers au moins un autre); et enfin le fait de ne pas partir (staying put) que van Hear considère, sans d'ailleurs 
réellement parvenir à convaincre le lecteur, comme faisant partie intégrante de la migration. Malgré cette dernière remarque, il démontre à partir de cette matrice que le mouvement migratoire ainsi décomposé ne peut être résumé par l'alternative volontaire-forcé : « Alors que le mouvement outward peut être contraint, précipité par la persécution, le conflit, la guerre ou toute autre circonstance menaçante pour la vie, le mouvement inward, y compris le choix de la destination, peut être façonné par des considérations économiques, matérielles ou les hasards de la vie » (p. 47). Ce modèle dynamique, qui veut lier les niveaux macro et micro dans une analyse plus concrète que celle des systèmes migratoires, s'appuie sur la création de plusieurs concepts dont celui d'ordre migratoire. Celui-ci est défini comme l'ensemble des «conditions, circonstances et de l'environnement dans le cadre duquel les migrants font des choix ou se voient imposés des choix» (p. 21). Cet ordre migratoire n'a de sens que localement, mais il est influencé selon van Hear par quatre domaines plus globaux: celui des causes profondes (économie macro-politique), celui des causes immédiates (des modifications structurelles de tout ordre: politique, économique, environnementale...), celui des «facteurs accélérateurs" (les crises, les catastrophes, les guerres) et enfin celui des facteurs d'appoint, ceux qui facilitent ou empêchent la migration : l'information, le transport, le régime migratoire international... Au total, l'analyse extraordinairement complète - parfois trop pour le lecteur - que propose van Hear sur ces dix crises migratoires, et qu'il fonde sur un gros travail de documentation et sur un travail de terrain au Yémen et en Jordanie pour lequel il a interviewé une centaine de migrants, cette analyse lui permet de mettre en évidence les logiques à l'oeuvre sur de nombreux aspects: politiques étatiques de persécution, décisions de migrer, accueil de ces populations dans d'autres pays et insertion socio-économique dans ces derniers. Au-delà de ces études de cas, ce que montre l'application du modèle de van Hear aux conséquences de ces crises, c'est que, considérant que les populations émigrées contraintes de migrer peuvent ensuite évoluer dans trois directions demeurer dans les pays d'accueil ; partir vers d'autres destinations; ou retourner dans le pays dont elles ont été expulsées - elles utilisent au moins deux de ces possibilités. En d'autres termes, ces crises migratoires peuvent être à la fois des moments de dédiasporisation par le retour de communautés migrantes vers leurs pays d'origine; de diasporisation par la dispersion vers d'autres pays d'une communauté migrante auparavant rassemblée ; et enfin de réaffirmation de la diaspora existante par le retour à l'ancien ordre migratoire. Bien plus que sa définition de la diaspora en tant que telle, c'est l'inscription dans l'histoire de l'analyse de van Hear, et son aptitude à prendre en compte les points de passage et les évolutions, qui en font un ouvrage stimulant et plein de promesses.

8 Au terme de ces lectures, deux pistes de recherche se profilent :

- Toute définition s'établit avant tout de façon négative, par différenciation avec des phénomènes proches mais non identiques. Pourtant, jusqu'à présent, si certaines définitions de la diaspora sont dissociées des communautés transnationales (van Hear) ou des communautés immigrées (Doomernik et van Amersfoort), c'est la plupart du temps de manière non systématique et à l'écart de tout comparatisme plus large. De plus, des aspects essentiels de la vie de ces populations émigrées sont passées sous silence, alors qu'ils pourraient être au fondement de définitions plus fines de l'organisation de l'expérience collective à l'étranger. Ainsi, la dimension politique, c'est-à-dire l'entretien d'activités politiques contre le régime en place dans le pays d'origine, disparait totalement des entreprises de définition de la diaspora, non parce 
qu'elle en est dissociée, mais parce qu'elle n'est pas considérée comme un facteur discriminant. C'est ainsi que Matjaz Klemencic peut signaler le fait que les émigrés "politiques" refusaient de se joindre aux "économiques" sans mettre en péril sa définition spontanée de la diaspora comme dispersion («The organization of the Slovène diaspora in the post World Bar II période and the policy of the Slovene and Yugoslav governments towards the Slovene diaspora ", p. 81-90). De la même manière, Robin Cohen peut consacrer de nombreuses pages à la question du foyer national dans la diaspora juive sans se demander si la politisation qui s'en est ensuivie n'a pas modifié une partie de cette diaspora pour la transformer en un autre mode d'organisation de l'expérience collective (p. 115-125). Enfin, comment peut-on justifier l'absence, dans toute étude sur la diaspora chinoise, des exilés anticommunistes en lutte contre Pékin ? Plutôt que de chercher à faire entrer la réalité sous la notion de diaspora, ou à l'en exclure, il est nécessaire de penser l'organisation de l'expérience collective à l'étranger en dehors de catégories substantialistes et figées. A la question «quoi ? (et rarement «qui ?») et « combien?»), il faut substituer la question «comment? ? pour restituer les processus par lesquels se forment les différents états de structuration de cette expérience. Car il s'agit bien de transformations d'état, de configurations dont la solidité réelle n'empêche nullement l'évolution ou le passage à un autre état. Pour ce faire, la sociologie constructiviste ${ }^{25}$ offre des pistes à condition de ne pas partir des dénominations, mais des processus, de faire l'inventaire des différences et des logiques pour, ensuite, puisqu'il le faut, leur donner un nom.

Stéphane DUFOIX Centre d'Analyse Comparative des Systèmes Politiques (Paris-I)

\section{NOTES}

1. L'utilisation postmodernisme de la notion de diaspora est particulièrement visible dans Chambers (I.), Migrancy, culture, identity, Londres, Routledge, 1994, et dans Hall (S.), " Cultural identity and diaspora », in Rutherford (J.), ed., Identity : community, culture, difference, Londres, Lawrence and Wishart, 1990.

2. Rosenau (J.), Turbulence in world politics, Princeton, Princeton University Press, 1990, ainsi que Badie (B.), La fin des territoires. Essai sur le désordre international et sur l'utilité sociale du respect, Paris, Fayard, 1995.

3. La présence du thème de la diaspora dans les études sur le nationalisme est variable. Il est absent de Hobsbawm (E.), Nations and nationalism since 1780, Cambridge, Cambridge University Press, 1990, et de Anderson (B.), L'imaginaire national : réflexions sur l'origine et l'essor du nationalisme, Paris, La Découverte, 1996 (1ère édition anglaise 1983) ; il est en revanche examiné chez Gellner (E.), Nations et nationalismes, Paris, Payot, 1989, et il est présent dans tous les travaux d'Anthony Smith : Nationalism in the 20th century, New York, New York University Press, 1979 ; The ethnic origins of nations, Oxford, Basil Blackwell, 1986, et Nations and nationalism in a global era, Cambridge, Polity Press, 1995.

4. Citons notamment Hovanessian (M.), « La notion de diaspora. Usages et champ sémantiques ", Journal des anthropologues, (72-73), 1998, p. 11-30, ainsi que Bilan des 
travaux sur la circularité migratoire, Rapport pour la Direction de la Population et des Migrations, novembre 1998. Signalons enfin qu'au mois de février 1999 fut diffusé sur France 3 le premier numéro (sur la diaspora irlandaise) d'une série de documentaires consacrés aux diasporas.

5. En tout pas moins de 14 diasporas différentes (grecque, juive, arménienne, indienne, slovène, assyro-chaldéenne, chinoise, bulgare, huguenote, latino-américaine, slave sudeuropéenne, turque, polonaise et libanaise). La diaspora grecque fait l'objet de six articles, dont un sur les Grecs Pontiques ; la diaspora juive de trois articles ; la diaspora slovène de deux articles comme la diaspora turque.

6. C'est le cas des articles de Yvo J.D. Peeters sur l'immigration et bilinguisme à Bruxelles, et de André-Louis Sanguin sur Montréal.

7. Parmi ces exceptions, citons Bassma Kodmani-Darwish, La diaspora palestinienne, Paris, PUF, 1997, ainsi que le texte introductif (non publié) de Dominique Schnapper à la Conférence Howard Gilman sur les diasporas et le transnationalisme, tenu à la Sorbonne du 18 au 20 novembre 1998. Quelques doctorants de science politique contribuent également à la réflexion française sur les diasporas : cf. Gilles Bertrand et Laurent Gayer, « Diasporas et " nouveaux conflits internationaux » » (à paraître prochainement dans Socio-logie de la paix).

8. Médam (A.), « Diaspora/Diasporas. Archétype et typologie », Revue européenne des migrations internationales, 9(1), 1993, p. 59-64.

9. Gérard Noiriel, «Qu'est-ce qu'une communauté immigrée ? ", in Pierre Milza et Denis Peschanski, eds., Italiens et Espagnols en France 1938-1946, Paris, IHTP, 1991, p. 309-312.

10. A l'exception notable des dix premières pages de la conclusion (p. 177-187), dont une intéressante analogie entre le jardinage et la typologie des diasporas.

11. Green (N.), «L'histoire comparative et le champ des études migratoires », Annales ESC, (6), novembre-décembre 1990, p. 1335-1350.

12. Gayer (L.), Diaspora indo-pakistanaise et régulation de la conflictualité à Londres et Paris, mémoire de DEA en science politique sous la direction de Bertrand Badie, IEP Paris, 1998.

13. Il n'existe à notre connaissance aucune étude précise ni sur les conditions d'utilisation scientifique du terme de " diaspora » ni sur l'évolution de l'autodésignation de ces « communautés transnationales » qui apparaît pourtant comme un point de départ indispensable. On trouvera quelques petits points de repère à propos des Juifs et des Arméniens dans Marienstras (R.), « Sur la notion de Diaspora », in Les minorités à l'âge de l'Etat-nation, Paris, Fayard, 1985, p. 215-226 ; Ter É (A.), « La diaspora arménienne », L’Espace géographique, (2), 1994, p. 115-128 ; ainsi que Hovanessian (M.), Territoires : mémoires et formes du croire ", Journal des anthropologues, (59), hiver 1995, p. 77-88. Si Riva Kastoryano estime que les premières utilisations du terme de diaspora remontent à 1967, Michel Bruneau montre que certains géographes français comme Max Sorre s'en servaient déjà en 1957. Cf. Bruneau (M.), « Espaces et territoires de diasporas », in Bruneau (M.), Diasporas, Montpellier, GIP-Reclus, 1995, p. 5-23.

14. Marienstras (R.), «Sur la notion de Diaspora », art.cit., p. 217-218.

15. Originellement publié sous le même titre dans L'espace géographique, (2), 1994, p. 106-113.

16. Lacoste (Y.), « Géopolitique des diasporas », Hérodote, (53), avril-juin 1989, p. 3-12. 
17. Sur la problématique sociologique des seuils et les erreurs qu'elle engendre, voir Granovetter (M.), « Threshold models of collective behavior », The American journal of sociology, 83(6), mai 1978, p. 1420-1443.

18. Sheffer (G.), ed., Modern diasporas in international politics, Londres, Croom Helm, 1986.

19. Pour une première présentation de ces deux éléments, cf Ma Mung (E.), « Dispositif économique et ressources spatiales : éléments d'une économie de diaspora ", Revue européenne des migrations internationales, 8(3), 1992, p. 175-194.

20. Outre l'énumération des éléments constitutifs d'une diaspora, Sheffer avait également, dès 1986, proposé une typologie les divisant entre « diasporas sans Etat » et « diasporas avec Etat », ces dernières se subdivisant en « classiques », " vétérans », « naissantes » et « dormantes ».

21. Safran (W.), « Diasporas in modern societies : myths of homeland and return », Diaspora, 1(1), 1991, p. 83-99.

22. Anthias (F.), « Evaluating « diaspora » : beyond ethnicity? », Sociology, 32(3), août 1998, p. 557-580.

23. Kay (D.), Chileans in exile. Private strategies, public lives, Edinburgh, Bechhoffer, 1987.

24. Voir notamment Richmond (A.), « Sociological theories of international migration : the case of refugees ", in Cohen (R.), ed., The sociology of migration, Cheltenham, Elgar, 1996, p. 331-349.

25. A l'exception notable du texte de Michèle Bouix dans RD, « Le refuge huguenot et les allégeances multiples ", p. 233-242, qui développe une perspective constructiviste et interpolaire fort intéressante, avant d'oublier la problématique qu'elle a forgée pour se cantonner dans la simple description des groupes huguenots en Angleterre, aux PaysBas et en Prusse.

INDEX

Mots-clés : Diaspora, migration 